\title{
Article \\ On the Conservation Laws and Exact Solutions to the (3+1)-Dimensional Modified KdV-Zakharov-Kuznetsov Equation
}

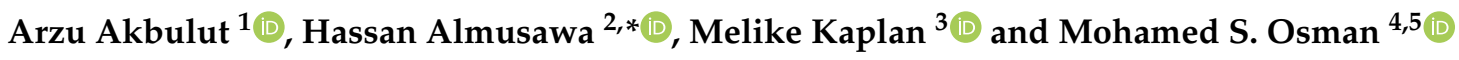 \\ 1 Department of Mathematics-Computer, Art-Science Faculty, Eskişehir Osmangazi University, \\ 26040 Eskişehir, Turkey; ayakut1987@hotmail.com \\ 2 Department of Mathematics, College of Sciences, Jazan University, Jazan 45142, Saudi Arabia \\ 3 Department of Mathematics, Art-Science Faculty, Kastamonu University, 37150 Kastamonu, Turkey; \\ mkaplan@kastamonu.edu.tr \\ 4 Department of Mathematics, Faculty of Science, Cairo University, Giza 12613, Egypt; mofatzi@sci.cu.edu.eg \\ 5 Department of Mathematics, Faculty of Applied Science, Umm Alqurah University, \\ Makkah 21955, Saudi Arabia \\ * Correspondence: haalmusawa@jazanu.edu.sa
}

check for updates

Citation: Akbulut, A.; Almusawa, A.; Kaplan, M.; Osman, M.S. On the Conservation Laws and Exact Solutions to the (3+1)-Dimensional Modified KdV-Zakharov-Kuznetsov Equation. Symmetry 2021, 13, 765. https://doi.org/10.3390/sym13050765

Academic Editor: Sun Young Cho

Received: 4 April 2021

Accepted: 27 April 2021

Published: 28 April 2021

Publisher's Note: MDPI stays neutral with regard to jurisdictional claims in published maps and institutional affiliations.

Copyright: (c) 2021 by the authors. Licensee MDPI, Basel, Switzerland. This article is an open access article distributed under the terms and conditions of the Creative Commons Attribution (CC BY) license (https:// creativecommons.org/licenses/by/ $4.0 /)$.

\begin{abstract}
In this paper, we consider conservation laws and exact solutions of the (3+1)-dimensional modified KdV-Zakharov-Kuznetsov equation. Firstly, we construct conservation laws of the given equation with the help of the conservation theorem; the developed conservation laws are modified conservation laws. Then, we obtain exact solutions of the given equation via the $\left(G^{\prime} / G, 1 / G\right)$ expansion method. The obtained solutions are classified as trigonometric solutions, hyperbolic solutions and rational solutions. Furthermore, graphical representations of the obtained solutions are given.
\end{abstract}

Keywords: conservation laws; partial differential equations; exact solutions; symbolic computation

\section{Introduction}

Many phenomena in physics and other fields can be modeled by nonlinear partial differential equations (NPDEs). When we want to understand the physical mechanism of natural phenomena described by NPDEs, we have to investigate the exact solutions of these equations [1-4]. Recently, a large number of methods that have been used to obtain exact solutions of NPDEs have become essential due to the existence of symbolic computation packages which aid researchers in the investigation of the dynamical behavior and the physical meaning of these solutions. Hirota's direct method [5-8], the tanh method [9], the exp-function method [10,11], $\left(G^{\prime} / G\right)$-expansion method [12,13], the extended $\left(G^{\prime} / G\right)$-expansion method [14,15], the extended trial equation method [16], the first integral method [17], the sine-cosine method [18] and the auxiliary equation method [19] are among these methods.

Conservation laws have a long history in physics. At the mathematical level, conservation laws are associated with the existence of a variational principle which admits symmetry transformations. This crucial fact was fully acknowledged by Emmy Noether. There are many different methods for the construction of conservation laws; for example, the characteristic method, variational approach, symmetry and conservation law relation, direct construction method, partial Noether approach, Noether approach, multiplier approach, conservation theorem and semi-implicit and semi-explicit methods [20-29]. In this work, we consider the conservation theorem.

The conservation theorem was introduced by Ibragimov. This theorem is associated with Lie symmetries and the formal Lagrangian of a given equation. We can construct the formal Lagrangian with the multiplication of the new adjoint variable and a given equation. 
Then, we can obtain an adjoint equation with the help of Euler-Lagrange equations. If we find the solution to the adjoint equation, we can obtain local conservation laws. Each Lie symmetry of a PDE yields a conservation law. These developed conservation laws can be trivial or non-trivial [30-35].

In this paper, we consider conservation laws and exact solutions of the $(3+1)$-dimensional modified KdV-Zakharov-Kuznetsov equation. We mention that in $(3+1)$-dimensional equations, multi-front solutions are described by their spatial structure and by their velocity composition. The spatial structure is actually composed of (3+1)-dimensional branes. Different clusters of fronts propagate at different velocities in certain branes, making them hybrids. Consequently, (3+1)-dimensional models show reasonable agreement with the movement of waves and demonstrate the potential to predict their location.

In Section 2, we have presented necessary information. Then, we give preliminaries about the conservation theorem in Section 2.1. We introduce the formal Lagrangian, adjoint equation and self-adjointness. Then, a general formula of conservation laws is given. Secondly, we present the $\left(G^{\prime} / G, 1 / G\right)$-expansion method in Section 2.2. In Section 3, we apply these methods to the (3+1)-dimensional modified KdV-Zakharov-Kuznetsov equation. In Section 3.1, we give admitted Lie point symmetries by the given equation; then, we construct the formal Lagrangian and adjoint equation. We find the solution to the adjoint equation to obtain the local conservation laws of the (3+1)-dimensional modified KdVZakharov-Kuznetsov equation. In Section 3.2, we obtain exact traveling wave solutions of the given equation. The obtained solutions are classified as trigonometric function solutions, hyperbolic function solutions and rational function solutions. In Section 4, conclusions are given.

\section{Fundamental Definitions and Mathematical Concepts}

\subsection{Information About Conservation Theorem}

We consider the $k$-th order system of partial differential equations in the following form:

$$
F_{\alpha}\left(x, u, u_{1}, u_{2}, \ldots, u_{k}\right)=0,
$$

where $x=\left(x_{1}, x_{2}, \ldots, x_{n}\right)$ are $n$ independent variables, $u=\left(u^{1}, u^{2}, \ldots, u^{m}\right)$ are $m$ dependent variables and $u_{j}$ is $j$-th order derivation respect to $x, j=1,2, \ldots, k$ and $\left.i_{j}=1,2, \ldots, n\right)$. Equation (1) admits the following infinitesimal generator:

$$
X=\xi^{i} \frac{\partial}{\partial x_{i}}+\eta^{\alpha} \frac{\partial}{\partial u^{\alpha}}
$$

where $\xi^{i}$ and $\eta^{\alpha}$ are infinitesimal functions, $i=1,2, \ldots, n$ and $\alpha=1,2, \ldots, m$. These functions contain only functions of dependent and independent variables. The $k$-th prolongation of the infinitesimal generator is

$$
X^{(k)}=X+\eta_{i}^{(1) \alpha} \frac{\partial}{\partial u_{i}^{\alpha}}+\ldots+\eta_{i_{1} i_{2} \ldots i_{k}}^{(k) \alpha} \frac{\partial}{\partial u_{i_{1} i_{2} \ldots i_{k}}^{\alpha}}, k \geq 1,
$$

where

$$
\begin{aligned}
\eta_{i}^{(1) \alpha} & =D_{i} \eta^{\alpha}-\left(D_{i} \xi^{j}\right) u_{j}^{\alpha} \\
\eta_{i_{1} i_{2} \ldots i_{k}}^{(k) \alpha} & =D_{i_{k}} \eta_{i_{1} i_{2} \ldots i_{k-1}}^{(k-1) \alpha}-\left(D_{i_{k}} \xi^{j}\right) u_{i_{1} i_{2} \ldots i_{k-1} j^{j}}^{\alpha}
\end{aligned}
$$

where $i, j=1,2, \ldots, n, \alpha=1,2, \ldots, m$ and $i_{l}=1,2, \ldots, m$ for $l=1,2, \ldots, k$ and $D_{i}$ is the total derivative operator with respect to $x_{i}$.

Not every PDE has a Lagrangian, but every PDE has a formal Lagrangian. This formal Lagrangian can be constructed with

$$
L=v^{\alpha} F_{\alpha} .
$$


The adjoint equation can be found with the help of Euler-Lagrange equations with following formula:

$$
F_{\alpha}^{*}\left(x, u, w, \ldots, u_{(k)}, w_{(k)}\right) \equiv \frac{\delta\left(v^{\alpha} F_{\alpha}\right)}{\delta u^{\alpha}}=0,
$$

where $\frac{\delta}{\delta u^{\alpha}}$ is variational derivative with

$$
\begin{aligned}
\frac{\delta}{\delta u^{\alpha}}= & \frac{\partial}{\partial u^{\alpha}}+\sum_{s=0}(-1)^{s} D_{i_{1}} \ldots D_{i_{s}} \frac{\partial}{\partial u_{i_{1} \ldots i_{s}}^{\alpha}} \\
& (\alpha=1, \ldots, m)
\end{aligned}
$$

The adjoint equation $F_{\alpha}^{*}$ admits symmetries of the original equation.

If we substitute the formal Lagrangian and infinitesimal functions in the following formula, we obtain $T^{i}$ conserved quantities:

$$
\begin{aligned}
T^{i}= & \xi^{i} L+W^{\alpha} \frac{\delta L}{\delta u_{i}^{\alpha}}+\sum_{s \geq 1} D_{i_{1}} \ldots D_{i_{s}}\left(W^{\alpha}\right) \frac{\partial}{\partial u_{i_{1} \ldots i_{s}}^{\alpha}}, \\
& (i=1, \ldots, n)
\end{aligned}
$$

where $W^{\alpha}=\eta^{\alpha}-\xi^{j} u_{j}^{\alpha}$ is the Lie characteristic function and $\alpha=1, \ldots, m$. The conserved quantities involve the arbitrary solutions of the adjoint equation, and thus one obtains an infinite number of conservation laws for Equation (1) by specifying $v^{\alpha}$.

If the conserved quantities satisfy the divergence condition

$$
D_{i}\left(T^{i}\right)=0,(i=1, \ldots, n),
$$

the conserved quantities are conservation laws of Equation (1) [36-38].

\subsection{Description of the $\left(G^{\prime} / G, 1 / G\right)$-Expansion Method}

In this subsection, we represent the $\left(G^{\prime} / G, 1 / G\right)$-expansion method to find the exact solutions of NPDEs step by step [39-41]. Firstly, some preparatory factors are presented.

Assume that we have a second-order linear ordinary differential equation as follows:

$$
G^{\prime \prime}(\xi)+\lambda G(\xi)=\mu,
$$

and we choose for simplicity

$$
\phi=G^{\prime} / G, \quad \gamma=1 / G
$$

From (8) and (9), we get

$$
\phi^{\prime}=-\phi^{2}+\mu \gamma-\lambda, \quad \gamma^{\prime}=-\phi \gamma
$$

Thus, we have three different cases for the general solution of Equation (8).

Case 1 (trigonometric function solutions) $\lambda>0$ : The general solution of Equation (8) is

$$
G(\xi)=C_{1} \sin (\sqrt{\lambda} \xi)+C_{2} \cos (\sqrt{\lambda} \xi)+\frac{\mu}{\lambda}
$$

and we have

$$
\gamma^{2}=\frac{\lambda}{\lambda^{2} \rho-\mu^{2}}\left(\phi^{2}-2 \mu \gamma+\lambda\right)
$$

where $C_{1}$ and $C_{2}$ are arbitrary constants and $\rho=C_{1}^{2}+C_{2}^{2}$. 
Case 2 (hyperbolic function solutions) $\lambda<0$ : The general solution of Equation (8) is

$$
G(\xi)=C_{1} \sinh (\sqrt{-\lambda} \xi)+C_{2} \cosh (\sqrt{-\lambda} \xi)+\frac{\mu}{\lambda}
$$

and we have

$$
\gamma^{2}=\frac{-\lambda}{\lambda^{2} \rho+\mu^{2}}\left(\phi^{2}-2 \mu \gamma+\lambda\right),
$$

where $C_{1}$ and $C_{2}$ are arbitrary constants and $\rho=C_{1}^{2}-C_{2}^{2}$.

Case 3 (rational function solutions) $\lambda=0$ : The general solution of Equation (8) is

$$
G(\xi)=\frac{\mu}{2} \xi^{2}+C_{1} \xi+C_{2}
$$

and we have

$$
\gamma^{2}=\frac{1}{C_{1}^{2}-2 \mu C_{2}}\left(\phi^{2}-2 \mu \gamma\right),
$$

where $C_{1}$ and $C_{2}$ are arbitrary constants.

Then, we suppose that we have an NPDE which is in the following form:

$$
P\left(u, u_{t}, u_{x}, u_{t t}, u_{x t}, u_{x x}, \ldots\right)=0
$$

where $x$ and $t$ are independent variables and (14) is a polynomial of $u$ and its partial derivatives. The main steps of the $\left(G^{\prime} / G, 1 / G\right)$-expansion method can be given as follows:

Step 1. By employing the traveling wave transformation $\xi=x+y+z-v t, u(x, y, z, t)=$ $u(\xi)$, Equation (14) is transformed into an ordinary differential equation:

$$
P\left(u,-v u^{\prime}, u^{\prime}, v^{2} u^{\prime \prime},-v u^{\prime}, u^{\prime \prime}, \ldots\right)=0
$$

Step 2. Then, we suppose that the exact solution of the Equation (15) can be expressed as follows:

$$
u(\xi)=\sum_{i=0}^{N} a_{i} \phi^{i}+\sum_{i=1}^{N} b_{i} \phi^{i-1} \gamma,
$$

where $G=G(\xi)$ satisfies Equation (8), $a_{i}(i=0, \ldots, N), b_{i}(i=1, \ldots, N), v, \lambda$ and $\mu$ are constants to be determined later. The positive integer $N$ can be found by considering the homogeneous balance principle between the highest-order derivatives and the nonlinear terms in Equation (15).

Step 3. Substituting (16) into Equation (15) along with Equations (10) and (11), (case 1 is taken as an example), the left-hand side of Equation (15) can be turned into a polynomial in $\phi$ and $\gamma$. We note that the degree of $\gamma$ is not larger than one. By equating each coefficient of this polynomial to zero, we can find an algebraic system of equations in terms of $a_{i}(i=0, \ldots, N), b_{i}(i=1, \ldots, N), v, \lambda(\lambda<0), \mu, C_{1}$ and $C_{2}$.

Step 4 . We solve the algebraic equations obtained in Step 3 with the aid of the Maple packet program. Then, by substituting the values of $a_{i}(i=0, \ldots, N), b_{i}(i=1, \ldots, N), v, \lambda, \mu$, $C_{1}$ and $C_{2}$ obtained into (16), the exact solutions of Equation (15), which is expressed by the hyperbolic functions, can be verified.

Step 5. Similar to Steps 3 and 4, substituting into Equation (16) into Equation (15) along with Equations (10) and (12) (or (10) and (13)), the exact solutions of Equation (15) are expressed by trigonometric functions (or by rational functions) and can be verified.

\section{Applications}

In this section, we apply the conservation theorem and $\left(G^{\prime} / G, 1 / G\right)$-expansion method to the $(3+1)$-dimensional modified KdV-Zakharov-Kuznetsov equation in the following form:

$$
u_{t}+\beta u^{2} u_{x}+u_{x x x}+u_{x y y}+u_{x z z}=0
$$


where $\beta$ is a nonzero arbitrary constant. This equation is an important class of NLEEs and arises in theoretical physics and plasma physics as well as fluid dynamics, quantum mechanics and nonlinear optics to analyze the basic properties of the nonlinear propagation of many physical phenomena, such as the amplitude and width of solitons, solitary wave structures and shock wave structures [42].

\subsection{Conservation Laws}

In this subsection, we use the conservation theorem to obtain conservation laws from Equation (17). The conservation theorem is deeply associated with Lie symmetry generators of PDEs, so we should find the Lie symmetry generators of Equation (17). Equation (17) admits the following six Lie symmetry generators (the set of $\left\{X_{1} ; X_{2} ; X_{3} ; X_{4} ; X_{5} ; X_{6}\right\}$ is closed under the Lie bracket):

$$
\begin{aligned}
& X_{1}=\frac{\partial}{\partial t}, X_{2}=\frac{\partial}{\partial y}, X_{3}=\frac{\partial}{\partial z^{\prime}}, \\
& X_{4}=\frac{\partial}{\partial x}, X_{5}=z \frac{\partial}{\partial y}-y \frac{\partial}{\partial z}, \\
& X_{6}=x \frac{\partial}{\partial x}+y \frac{\partial}{\partial y}+z \frac{\partial}{\partial z}+3 t \frac{\partial}{\partial t}-u \frac{\partial}{\partial u} .
\end{aligned}
$$

Now, we can obtain the formal Lagrangian of the given equation by

$$
L=v\left(u_{t}+\beta u^{2} u_{x}+u_{x x x}+u_{x y y}+u_{x z z}\right)
$$

where $v$ is the adjoint variable. The adjoint equation can be calculated with Formula (5) and Equation (18) as

$$
F^{*}=-\beta u^{2} v_{x}-v_{t}-v_{x x x}-v_{x y y}-v_{x z z}=0 .
$$

We can see that the adjoint equation is self-adjoint. $v=1$ and $v=u$ are solutions of the adjoint equation. The special form of the conserved quantities formula for Equation (17) is given by

$$
\begin{gathered}
T^{x}=\quad L \xi^{x}+W\left[\frac{\partial L}{\partial u_{x}}+D_{x}^{2}\left(\frac{\partial L}{\partial u_{x x x}}\right)+D_{y}^{2}\left(\frac{\partial L}{\partial u_{x y y}}\right)+D_{z}^{2}\left(\frac{\partial L}{\partial u_{x z z}}\right)\right] \\
+D_{x}(W)\left[-D_{x}\left(\frac{\partial L}{\partial u_{x x x}}\right)\right]+D_{y}(W)\left[-D_{y}\left(\frac{\partial L}{\partial u_{x y y}}\right)\right] \\
+D_{z}(W)\left[-D_{z}\left(\frac{\partial L}{\partial u_{x z z}}\right)\right] \\
+D_{x}^{2}(W)\left[\frac{\partial L}{\partial u_{x x x}}\right]+D_{y}^{2}(W)\left[\frac{\partial L}{\partial u_{x y y}}\right]+D_{z}^{2}(W)\left[\frac{\partial L}{\partial u_{x z z}}\right] \\
T^{y}=L \xi^{y}+W\left[D_{x y}^{2}\left(\frac{\partial L}{\partial u_{x y y}}\right)\right]+D_{x}(W)\left[-D_{y}\left(\frac{\partial L}{\partial u_{x y y}}\right)\right] \\
+D_{y}(W)\left[-D_{x}\left(\frac{\partial L}{\partial u_{x y y}}\right)\right]+D_{x y}(W)\left[\frac{\partial L}{\partial u_{x y y}}\right]
\end{gathered}
$$




$$
\begin{aligned}
T^{z}= & L \xi^{z}+W\left[D_{x z}^{2}\left(\frac{\partial L}{\partial u_{x z z}}\right)\right]+D_{x}(W)\left[-D_{z}\left(\frac{\partial L}{\partial u_{x z z}}\right)\right] \\
& +D_{z}(W)\left[-D_{x}\left(\frac{\partial L}{\partial u_{x z z}}\right)\right]+D_{x z}(W)\left[\frac{\partial L}{\partial u_{x z z}}\right]
\end{aligned}
$$

and

$$
T^{t}=L \xi^{t}+W\left[\frac{\partial L}{\partial u_{t}}\right] .
$$

Each Lie point symmetry of the given equation gives a conservation law, so we can construct 12 local conservation laws with $v=1$ and $v=u$.

Case 1: In this case, we use $v=1$ to obtain the conservation laws of Equation (17).

(1.1) When $X_{1}=\frac{\partial}{\partial t}$, we obtain the following conserved quantities:

$$
\begin{aligned}
& T_{1,1}^{x}=-\beta u^{2} u_{t}-u_{x x t}-u_{y y t}-u_{z z t}, \\
& T_{1,1}^{y}=-u_{x y t}, \\
& T_{1,1}^{z}=-u_{x z t}, \\
& T_{1,1}^{t}=-u_{t} .
\end{aligned}
$$

If these conserved quantities satisfy the divergence condition, they are trivial conservation laws; otherwise, we must adjust the conserved quantities. In (24), the conserved quantities do not satisfy the divergence condition.

$$
D_{x}\left(T_{1,1}^{x}\right)+D_{y}\left(T_{1,1}^{y}\right)+D_{z}\left(T_{1,1}^{z}\right)+D_{t}\left(T_{1,1}^{t}\right)=D_{x}\left(-u_{y y t}-u_{z z t}\right)
$$

Thus, the modified conservation laws are

$$
\begin{aligned}
\widetilde{\sim} & =-\beta u^{2} u_{t}-u_{x x t}, \\
T_{1,1}^{x} & =-u_{x y t}, \\
T_{1,1}^{y} & =-u_{x z t}, \\
T_{1,1}^{z} & =-u_{t} . \\
\underset{T_{1,1}^{t}}{z} & =
\end{aligned}
$$

(1.2) When $X_{2}=\frac{\partial}{\partial y}$, the conserved quantities are

$$
\begin{aligned}
& T_{1,2}^{x}=-\beta u^{2} u_{y}-u_{x x y}-u_{y y y}-u_{y z z}, \\
& T_{1,2}^{y}=u_{t}+\beta u^{2} u_{x}+u_{x x x}+u_{x z z}, \\
& T_{1,2}^{z}=-u_{x y z} \\
& T_{1,2}^{t}=-u_{y} .
\end{aligned}
$$

The divergence condition $D_{i}\left(T_{i}\right)$ is not equal to zero, but the divergence condition can be written under the total derivative:

$$
D_{x}\left(T_{1,2}^{x}\right)+D_{y}\left(T_{1,2}^{y}\right)+D_{z}\left(T_{1,2}^{z}\right)+D_{t}\left(T_{1,2}^{t}\right)=D_{x}\left(-u_{y y y}-u_{y z z}\right)
$$

The nontrivial conservation laws with some adjustment are given by

$$
\begin{aligned}
\underset{T_{1,2}^{x}}{\sim} & =-\beta u^{2} u_{y}-u_{x x y}, \\
\underset{\sim}{T_{1,2}^{y}} & =-u_{x y y}, \\
\underset{T_{1,2}^{z}}{\tilde{r}} & =-u_{x y z}, \\
\underset{1,2}{t} & =-u_{y} .
\end{aligned}
$$


(1.3) If we use $X_{3}=\frac{\partial}{\partial z}$, we obtain $W=-u_{z}$; then, the conserved quantities are

$$
\begin{aligned}
& T_{1,3}^{x}=-\beta u^{2} u_{z}-u_{x x z}-u_{y y z}-u_{z z z} \\
& T_{1,3}^{y}=-u_{x y z} \\
& T_{1,3}^{z}=u_{t}+\beta u^{2} u_{x}+u_{x x x}+u_{x y y} \\
& T_{1,3}^{t}=u_{t}+\beta u^{2} u_{x}+u_{x x x}+u_{x y y}+u_{x z z}-u_{z} .
\end{aligned}
$$

The divergence condition $D_{i}\left(T_{i}\right)$ is different from zero. The divergence condition can be written under the total derivative with

$$
D_{x}\left(T_{1,3}^{x}\right)+D_{y}\left(T_{1,3}^{y}\right)+D_{z}\left(T_{1,3}^{z}\right)+D_{t}\left(T_{1,3}^{t}\right)=D_{x}\left(-u_{y y z}-u_{z z z}\right) .
$$

Then, the modified conservation laws are

$$
\begin{aligned}
\widetilde{\widetilde{T}} & \underset{1,3}{x}=-\beta u^{2} u_{z}-u_{x x z}, \\
T_{1,3}^{y} & =-u_{x y z}, \\
\widetilde{T_{1,3}} & =-u_{x z z} \\
\widetilde{T_{1,3}^{z}} & =-u_{z} .
\end{aligned}
$$

(1.4) When $X_{4}=\frac{\partial}{\partial x}$, the found conserved quantities are given by

$$
\begin{aligned}
& T_{1,4}^{x}=u_{t}, \\
& T_{1,4}^{y}=-u_{x x y}, \\
& T_{1,4}^{z}=-u_{x x z} \\
& T_{1,4}^{t}=-u_{x} .
\end{aligned}
$$

These conserved quantities do not satisfy the divergence condition in Equation (7). We can write the divergence condition with

$$
D_{x}\left(T_{1,4}^{x}\right)+D_{y}\left(T_{1,4}^{y}\right)+D_{z}\left(T_{1,4}^{z}\right)+D_{t}\left(T_{1,4}^{t}\right)=D_{y}\left(-u_{x x y}\right)+D_{z}\left(-u_{x x z}\right) .
$$

Then, the found, modified conservation laws are

$$
\widetilde{T_{1,4}^{x}}=u_{t}, \widetilde{T_{1,4}^{t}}=-u_{x}, \widetilde{T_{1,4}^{y}}=\widetilde{T_{1,4}^{z}}=0 .
$$

(1.5) If we use $X_{5}=z \frac{\partial}{\partial y}-y \frac{\partial}{\partial z}$, the obtained conserved quantities are

$$
\begin{aligned}
T_{1,5}^{x}= & -\beta u^{2} u_{y} z+\beta u^{2} u_{z} y-u_{x x y} z+u_{x x z} y, \\
& -u_{y y y} z+u_{y y z} y-u_{z z y} z+u_{z z z} y \\
T_{1,5}^{y}= & z u_{t}+\beta u^{2} u_{x} z+u_{x x x} z+u_{x z z} z+u_{x y z} y+u_{x z}, \\
T_{1,5}^{z}= & -y u_{t}-\beta u^{2} u_{x} y-y u_{x x x}-y u_{x y y}-z u_{x y z}-u_{x y} \\
T_{1,5}^{t}= & -y u_{t}-y \beta u^{2} u_{x}-y u_{x x x}-y u_{x y y}-y u_{x z z}-z u_{y}+y u_{z} .
\end{aligned}
$$

We can see that the divergence condition $D_{i}\left(T_{i}\right)$ is not equal to zero, but this condition can be written under the total derivative with

$$
D_{x}\left(T_{1,5}^{x}\right)+D_{y}\left(T_{1,5}^{y}\right)+D_{z}\left(T_{1,5}^{z}\right)+D_{t}\left(T_{1,5}^{t}\right)=D_{x}\left(-z u_{y y y}-z u_{z z y}+y u_{y y z}+y u_{z z z}\right) .
$$


The modified conservation laws are

$$
\begin{aligned}
\underset{T_{1,5}^{\sim}}{\sim} & =-\beta u^{2} u_{y} z+\beta u^{2} u_{z} y-u_{x x y} z+u_{x x z} y, \\
T_{1,5}^{y} & =-u_{x y y} z+u_{x y z} y+u_{x z} \\
T_{1,5}^{z} & =y u_{x z z}-z u_{x y z}-u_{x y} \\
T_{1,5}^{t} & =-z u_{y}+y u_{z} .
\end{aligned}
$$

(1.6) When $X_{6}=x \frac{\partial}{\partial x}+y \frac{\partial}{\partial y}+z \frac{\partial}{\partial z}+3 t \frac{\partial}{\partial t}-u \frac{\partial}{\partial u}$, we get $W=-u-x u_{x}-y u_{y}-z u_{z}-$ $3 t u_{t}$. From Equations (20)-(23), we obtain the conservation laws as follows:

$$
\begin{aligned}
T_{1,6}^{x}= & -z\left(\beta u^{2} u_{z}+u_{x x z}+u_{y y z}+u_{z z z}\right)-3 u_{x x} \\
& -y\left(\beta u^{2} u_{y}+u_{x x y}+u_{y y y}+u_{z z y}\right)-3 u_{y y}-\beta u^{3} \\
& -3 t\left(\beta u^{2} u_{t}+u_{x x t}+u_{y y t}+u_{z z t}\right)-3 u_{z z}+x u_{t} \\
T_{1,6}^{y}= & y\left(u_{t}+\beta u^{2} u_{x}+u_{x x x}+u_{x z z}\right)-3 u_{x y}-x u_{x x y}-z u_{x y z}-3 t u_{x y t}, \\
T_{1,6}^{z}= & z\left(u_{t}+\beta u^{2} u_{x}+u_{x x x}+u_{x y y}\right)-3 u_{x z}-x u_{x x z}-y u_{x y z}-3 t u_{x z t}, \\
T_{1,6}^{t}= & z\left(u_{t}+\beta u^{2} u_{x}+u_{x x x}+u_{x y y}\right)+z u_{x z z}-u-x u_{x}-y u_{y}-z u_{z}-3 t u_{t} .
\end{aligned}
$$

The divergence condition $D_{i}\left(T_{i}\right)$ is not equal to zero and includes extra terms. These terms can be absorbed as

$$
\begin{aligned}
D_{x}\left(T_{1,6}^{x}\right)+D_{y}\left(T_{1,6}^{y}\right)+D_{z}\left(T_{1,6}^{z}\right)+D_{t}\left(T_{1,6}^{t}\right)= & D_{t}\left(-3 t u_{x z z}-3 t u_{x y y}\right) \\
& +D_{y}\left(-x u_{x x y}-y u_{x y y}-z u_{x y z}\right) \\
& +D_{z}\left(-x u_{x x z}-y u_{x y z}-z u_{x z z}\right) .
\end{aligned}
$$

When $v=1$, we obtain the following non-trivial conservation laws:

$$
\begin{aligned}
\underset{1,6}{T_{1,6}^{x}} & =T_{1,6}^{x} \\
\underset{T_{1,6}^{y}}{\sim} & =-3 u_{x y}-3 t u_{x y t}, \\
T_{1,6}^{z} & =-3 u_{x z}-3 t u_{x z t} \\
\underset{T_{1,6}^{t}}{t} & =-u-x u_{x}-y u_{y}-z u_{z}-3 t\left(u_{t}-u_{x z z}-u_{x y y}\right)
\end{aligned}
$$

Case 2: In this case, we use $v=u$ to obtain the conservation laws of Equation (17). vectors:

(2.1) Using the symmetry generator $X_{1}=\frac{\partial}{\partial t}$, we obtain the following conserved

$$
\begin{aligned}
T_{2,1}^{x}= & -u_{t}\left(\beta u^{3}+u_{x x}+u_{y y}+u_{z z}\right)+u_{x} u_{x t} \\
& -u\left(u_{x x t}+u_{y y t}+u_{z z t}\right)+u_{z} u_{z t}+u_{y} u_{y t}, \\
T_{2,1}^{y}= & -u_{t} u_{x x}+u_{y} u_{x t}+u_{x} u_{y t}-u u_{x y t}, \\
T_{2,1}^{z}= & -u_{t} u_{x x}+u_{z} u_{x t}+u_{x} u_{z t}-u u_{x z t}, \\
T_{2,1}^{t^{\prime}}= & -u u_{t} .
\end{aligned}
$$

The divergence condition is different from zero.

$$
\begin{aligned}
\sum_{i=1}^{4} D_{i}\left(T_{2,1}^{i}\right)= & D_{x}\left(-u u_{y y t}-u u_{z z t}\right)+D_{y}\left(-u_{t} u_{x x}+u_{x} u_{t y}\right) \\
& +D_{z}\left(u_{x} u_{z t}-u_{t} u_{x x}\right)+D_{t}\left(u_{z} u_{x z}+u_{y} u_{x y}\right)
\end{aligned}
$$

The modified conservation laws with the help of (43) are 


$$
\begin{aligned}
\underset{T_{2,1}^{x}}{\widetilde{x}}=T_{2,1}^{x}+u u_{y y t}+u u_{z z t}, \\
T_{2,1}^{y}=T_{2,1}^{y}+u_{t} u_{x x}-u_{x} u_{t y}, \\
\underset{\widetilde{\sim}}{T_{2,1}^{z}}=T_{2,1}^{z}-u_{x} u_{z t}+u_{t} u_{x x} \\
\widetilde{T_{2,1}^{t}}=T_{2,1}^{t}-u_{z} u_{x z}-u_{y} u_{x y} .
\end{aligned}
$$

(2.2) When $X_{2}=\frac{\partial}{\partial y}$, the conserved quantities are

$$
\begin{aligned}
T_{2,2}^{x}= & -u_{y}\left(\beta u^{3}+u_{x x}+u_{z z}\right)+u_{x} u_{x y}+u_{z} u_{y z} \\
& -u\left(u_{x x y}+u_{y y y}+u_{z z y}\right) \\
T_{2,2}^{y}= & u\left(u_{t}+\beta u^{2} u_{x}+u_{x x x}+u_{x z z}\right)-u_{y} u_{x x}+u_{y} u_{x y}+u_{x} u_{y y}, \\
T_{2,2}^{z}= & -u_{y} u_{x x}+u_{z} u_{x y}+u_{x} u_{z y}-u u_{x y z} \\
T_{2,2}^{t}= & -u u_{y} .
\end{aligned}
$$

The divergence condition can be expressed for these conserved vectors as follows:

$$
\begin{aligned}
\sum_{i=1}^{4} D_{i}\left(T_{2,2}^{i}\right)= & D_{x}\left(-u u_{y y y}-u u_{y z z}\right)+D_{z}\left(-u_{y} u_{x x}+u_{x} u_{y z}\right) \\
& +D_{y}\left(u_{y} u_{x y}+u_{z} u_{x z}-u_{y} u_{x x}+u_{x} u_{y y}\right) .
\end{aligned}
$$

Then, the modified conservation laws are given by

$$
\begin{aligned}
\underset{T_{2,2}^{x}}{\tilde{x}} & =T_{2,2}^{x}+u u_{y y y}+u u_{y z z} \\
T_{2,2}^{\tilde{y}} & =-u u_{x y y}-u_{z} u_{x z} \\
T_{2,2}^{z} & =u_{z} u_{x y}-u u_{x y z} \\
T_{2,2}^{t} & =-u u_{y} .
\end{aligned}
$$

(2.3) Using the symmetry generator $X_{3}=\frac{\partial}{\partial z}$, we obtain the following conserved vectors:

$$
\begin{aligned}
T_{2,3}^{x}= & -u_{z}\left(\beta u^{3}+u_{x x}+u_{y y}\right)+u_{x} u_{x z}+u_{y} u_{y z} \\
& -u\left(u_{x x z}+u_{y y z}+u_{z z z}\right) \\
T_{2,3}^{y}= & -u_{z} u_{x x}+u_{y} u_{x z}+u_{x} u_{z y}-u u_{x y z} \\
T_{2,3}^{z}= & u\left(u_{t}+\beta u^{2} u_{x}+u_{x x x}+u_{x y y}\right)-u_{z} u_{x x}+u_{z} u_{x z}+u_{x} u_{z z} \\
T_{2,3}^{t}= & u\left(u_{t}+\beta u^{2} u_{x}+u_{x x x}+u_{x y y}+u_{x z z}\right)-u u_{z} .
\end{aligned}
$$

We can see that the divergence condition $D_{i}\left(T_{i}\right)$ is not equal to zero as follows:

$$
\begin{aligned}
\sum_{i=1}^{4} D_{i}\left(T_{2,3}^{i}\right)= & D_{x}\left(-u u_{y y z}\right)+D_{y}\left(-u_{z} u_{x x}+u_{x} u_{y z}\right) \\
& +D_{z}\left(u_{y} u_{x y}+u_{z} u_{x z}-u_{z} u_{x x}+u_{z} u_{x z}-u u_{x z z}\right) .
\end{aligned}
$$

The modified conservation laws are

$$
\begin{aligned}
\underset{T_{2,3}^{x}}{T_{2,3}^{x}} & =T_{2,3}^{x}+u u_{y y z}, \\
T_{2,3}^{y} & =u_{x z} u_{y}-u u_{x y z}, \\
T_{2,3}^{z} & =u_{z z} u_{x}-u_{y} u_{x y}-u_{z} u_{x z}, \\
T_{2,3}^{t} & =-u u_{z} .
\end{aligned}
$$


(2.4) When $X_{4}=\frac{\partial}{\partial x}$, the found conserved quantities are given by

$$
\begin{aligned}
T_{2,4}^{x} & =u u_{t}-u_{x} u_{y y}-u_{x} u_{z z}+u_{x y} u_{y}+u_{x z} u_{z} \\
T_{2,4}^{y} & =-u_{x} u_{x x}+u_{y} u_{x x}+u_{x} u_{x y}-u u_{x x y} \\
T_{2,4}^{z} & =-u_{x} u_{x x}+u_{z} u_{x x}+u_{x} u_{x z}-u u_{x x z} \\
T_{2,4}^{t} & =-u u_{x} .
\end{aligned}
$$

These conserved quantities do not satisfy the divergence condition in (7). We can write the divergence condition with

$$
\begin{aligned}
\sum_{i=1}^{4} D_{i}\left(T_{2,4}^{i}\right)= & D_{z}\left(-u u_{x x z}\right)+D_{y}\left(-u u_{x x y}\right) \\
& +D_{x}\left(2 u_{y} u_{x y}+2 u_{z} u_{x z}-u_{x} u_{x z}-u_{x} u_{x y}\right) .
\end{aligned}
$$

The modified conservation laws are constructed with the help of (52) as

$$
\begin{aligned}
\underset{T_{2,4}^{x}}{\widetilde{x}} & =u u_{t}-u_{x} u_{y y}-u_{x} u_{z z}-u_{x y} u_{y}-u_{x z} u_{z}+u_{x} u_{x z}+u_{x} u_{x y} \\
T_{2,4}^{y} & =-u_{x} u_{x x}+u_{y} u_{x x}+u_{x} u_{x y} \\
\underset{T_{2,4}^{z}}{\widetilde{y}} & =-u_{x} u_{x x}+u_{z} u_{x x}+u_{x} u_{x z} \\
\widetilde{T_{2,4}^{t}} & =-u u_{x} .
\end{aligned}
$$
are

(2.5) If we use $X_{5}=z \frac{\partial}{\partial y}-y \frac{\partial}{\partial z}$ and Equation (18), the obtained conserved quantities

$$
\begin{aligned}
T_{2,5}^{x}= & -z u_{y}\left(\beta u^{3}+u_{x x}+u_{z z}\right)+u y\left(u_{y y z}+u_{z z z}+u_{x x z}\right) \\
& +y u_{z}\left(\beta u^{3}+u_{x x}+u_{y y}\right)-z u\left(u_{x x y}+u_{y y y}+u_{y z z}\right) \\
& +z u_{x} u_{x y}+z u_{z} u_{z y}-y u_{x} u_{x z}-y u_{y} u_{z y}, \\
T_{2,5}^{y}= & u z\left(u_{t}+\beta u^{2} u_{x}+u_{x x x}+u_{x z z}\right)-z u_{y}\left(u_{x x}-u_{x y}\right)+u u_{x z} \\
& +y u_{z} u_{x x}-y u_{y} u_{x z}+z u_{x} u_{y y}-y u_{x} u_{z y}-u_{x} u_{z}+y u u_{x y z \prime} \\
T_{2,5}^{z}= & -u y\left(u_{t}+\beta u^{2} u_{x}+u_{x x x}+u_{x y y}\right)-u\left(z u_{x y z}+u_{x y}\right) \\
& y u_{z}\left(u_{x x}-u_{x z}\right)+u_{x}\left(u_{y}+z u_{y z}-y u_{z z}\right)-z u_{y} u_{x x}+z u_{z} u_{x y}, \\
T_{2,5}^{t}= & -u y\left(u_{t}+\beta u^{2} u_{x}+u_{x x x}+u_{x y y}+u_{x z z}\right)-u z u_{y}+y u u_{z} .
\end{aligned}
$$

The divergence condition can be written under the total derivative as

$$
\begin{aligned}
\sum_{i=1}^{4} D_{i}\left(T_{2,5}^{i}\right)= & D_{x}\left(z u_{z} u_{z y}-2 y u_{z} u_{z z}+2 z u_{y} u_{y y}-z u u_{y z z}-y u_{y} u_{y z}+u_{y} u_{z}\right) \\
& +D_{y}\left(-z u u_{x y y}-z u_{y} u_{x x}+y u_{z} u_{x x}-y u_{x y} u_{z}-u_{x} u_{z}\right) \\
& +D_{z}\left(u y u_{x z z}+u y u_{x y y}-z u_{y} u_{x x}+y u_{x x} u_{z}+z u_{x} u_{z y}\right) .
\end{aligned}
$$

The modified conservation laws can be written with

$$
\begin{aligned}
\underset{\widetilde{\sim}}{T_{2,5}^{x}}=T_{2,5}^{x}-z u_{z} u_{z y}+2 y u_{z} u_{z z}-2 z u_{y} u_{y y}+z u u_{y z z}+y u_{y} u_{y z}-u_{y} u_{z} \\
T_{2,5}^{y}=T_{2,5}^{y}+z u u_{x y y}+z u_{y} u_{x x}-y u_{z} u_{x x}+y u_{x y} u_{z}+u_{x} u_{z} \\
T_{2,5}^{z}=T_{2,5}^{z}-u y u_{x z z}-u y u_{x y y}+z u_{y} u_{x x}-y u_{x x} u_{z}-z u_{x} u_{z y}, \\
T_{2,5}^{t}=-u z u_{y}+y u u_{z} .
\end{aligned}
$$

\subsection{Exact Solutions}

The traveling wave transformation

$$
u(x, y, z, t)=u(\xi), \xi=x+y+z-v t,
$$

transforms Equation (17) to the following ordinary differential equation: 


$$
-v u^{\prime}+\beta u^{2} u^{\prime}+3 u^{\prime \prime \prime}=0 .
$$

By integrating Equation (58) with respect to $\xi$ once, we obtain

$$
-v u+\beta \frac{u^{3}}{3}+3 u^{\prime \prime}+C=0 .
$$

where $C$ is the integration constant. Balancing the highest-order derivative $u^{\prime \prime}$ and the nonlinear term $u^{3}$ from Equation (59), yields $3 N=N+2$ which gives $N=1$. So, Equation (16) takes the following form

$$
u(\xi)=a_{0}+a_{1} \phi+b_{1} \gamma,
$$

where $a_{0}, a_{1}$ and $b_{1}$ are constants to be determined.

Case 1 (Trigonometric function solutions) $\lambda>0$.

With the aid of symbolic computation, substituting Equation (60) into Equation (59) along with Equations (10) and (11) and collecting coefficients of polynomials of $\phi^{i} \gamma^{j}(i, j=$ $0,1,2,3)$, then we find an algebraic system of $\rho, \mu, \lambda, \beta, a_{0}, a_{1}, b_{0}$ as follows:

$$
\begin{aligned}
\phi^{3}: & \frac{\beta a_{1}^{3} \lambda^{4} \rho^{2}+\beta a_{1}^{3} \mu^{4}-2 \beta a_{1}^{3} \lambda^{2} \rho \mu^{2}}{3}-12 a_{1} \lambda^{2} \rho \mu^{2}+6 a_{1} \mu^{4} \\
& +\beta a_{1} b_{1}^{2} \lambda^{3} \rho-\beta a_{1} b_{1}^{2} \lambda \mu^{2}+6 a_{1} \lambda^{4} \rho^{2}=0, \\
\phi^{2} \gamma^{1}:= & \beta a_{1}^{2} b_{1} \lambda^{4} \rho^{2}-12 b_{1} \lambda^{2} \rho \mu^{2}+\frac{\beta b_{1}^{3} \lambda^{3} \rho-\beta b_{1}^{3} \lambda \mu^{2}}{3}+6 b_{1} \lambda^{4} \rho^{2} \\
& +\beta a_{1}^{2} b_{1} \mu^{4}+6 b_{1} \mu^{4}-2 \beta a_{1}^{2} b_{1} \lambda^{2} \rho \mu^{2}=0, \\
\phi^{2} \gamma^{0}: \quad & \beta a_{0} b_{1}^{2} \lambda^{3} \rho-2 \beta a_{0} a_{1}^{2} \lambda^{2} \rho \mu^{2}+\beta a_{0} a_{1}^{2} \lambda^{4} \rho^{2}-3 b_{1} \mu \lambda^{3} \rho \\
& +3 b_{1} \mu^{3} \lambda-\beta a_{0} b_{1}^{2} \lambda \mu^{2}-\frac{2}{3} \beta b_{1}^{3} \lambda^{2} \mu+\beta a_{0} a_{1}^{2} \mu^{4}=0, \\
\phi^{1} \gamma^{1}:= & 2 \beta a_{0} a_{1} b_{1} \lambda^{4} \rho^{2}-2 \beta a_{1} b_{1}^{2} \lambda^{3} \mu \rho-9 a_{1} \mu \lambda^{4} \rho^{2}+2 \beta a_{1} b_{1}^{2} \lambda \mu^{3} \\
& +18 a_{1} \mu^{3} \lambda^{2} \rho+2 \beta a_{0} a_{1} b_{1} \mu^{4}-4 \beta a_{0} a_{1} b_{1} \lambda^{2} \rho \mu^{2}-9 a_{1} \mu^{5}=0, \\
\phi^{1} \gamma^{0}: \quad & -v a_{1} \mu^{4}-12 a_{1} \lambda^{3} \rho \mu^{2}-v a_{1} \lambda^{4} \rho^{2}+\beta a_{0}^{2} a_{1} \lambda^{4} \rho^{2}-2 \beta a_{0}^{2} a_{1} \lambda^{2} \rho \mu^{2}+6 a_{1} \lambda^{5} \rho^{2} \\
& -\beta a_{1} b_{1}^{2} \lambda^{2} \mu^{2}+6 a_{1} \lambda \mu^{4}+\beta a_{1} b_{1}^{2} \lambda^{4} \rho+2 v a_{1} \lambda^{2} \rho \mu^{2}+\beta a_{0}^{2} a_{1} \mu^{4}=0, \\
\phi^{0} \gamma^{1}: \quad & -2 \beta a_{0}^{2} b_{1} \lambda^{2} \rho \mu^{2}-v b_{1} \mu^{4}+3 b_{1} \lambda^{5} \rho^{2}-3 b_{1} \lambda \mu^{4}-2 \beta a_{0} b_{1}^{2} \lambda^{3} \mu \rho-v b_{1} \lambda^{4} \rho^{2} \\
& +\beta a_{0}^{2} b_{1} \mu^{4}+\beta b_{1}^{3} \lambda^{2} \mu^{2}+\frac{\beta b_{1}^{3} \lambda^{4} \rho}{3}+2 \beta a_{0} b_{1}^{2} \lambda \mu^{3}+\beta a_{0}^{2} b_{1} \lambda^{4} \rho^{2}+2 v b_{1} \lambda^{2} \rho \mu^{2}=0, \\
\phi^{0} \gamma^{0}: \quad & C \lambda^{4} \rho^{2}+\frac{\beta a_{0}^{3} \mu^{4}+\beta a_{0}^{3} \lambda^{4} \rho^{2}-2 \beta b_{1}^{3} \lambda^{3} \mu-2 \beta a_{0}^{3} \lambda^{2} \rho \mu^{2}}{3}+3 b_{1} \mu^{3} \lambda^{2}-v a_{0} \mu^{4}+2 v a_{0} \lambda^{2} \rho \mu^{2} \\
& +C \mu^{4}-2 C \lambda^{2} \rho \mu^{2}-3 b_{1} \mu \lambda^{4} \rho-v a_{0} \lambda^{4} \rho^{2}+\beta a_{0} b_{1}^{2} \lambda^{4} \rho-\beta a_{0} b_{1}^{2} \lambda^{2} \mu^{2}=0
\end{aligned}
$$

The solution of this system gives

$$
\begin{array}{clrl}
a_{0} & =\mp \frac{9 \mu}{\beta \sqrt{\frac{18\left(\mu^{2}-\lambda^{2} \rho\right)}{\beta \lambda}}}, \quad a_{1}=0, & b_{1}= \pm \sqrt{\frac{18\left(\mu^{2}-\lambda^{2} \rho\right)}{\beta \lambda}}, \\
v=\frac{3 \lambda\left(\mu^{2}+2 \lambda^{2} \rho\right)}{2\left(\mu^{2}-\lambda^{2} \rho\right)}, & C= \pm \frac{27 \lambda^{3} \rho \mu}{\left(\mu^{2}-\lambda^{2} \rho\right) \beta \sqrt{\frac{18\left(\mu^{2}-\lambda^{2} \rho\right)}{\beta \lambda}}} .
\end{array}
$$

If we substitute these results into Equation (60) along with Equations (9) and (11), we obtain the periodic solutions of the (3+1)-dimensional modified KdV-Zakharov-Kuznetsov equation as follows:

$$
u(\xi)=\mp \frac{9 \mu}{\beta \sqrt{\frac{18\left(\mu^{2}-\lambda^{2}\left(C_{1}^{2}+C_{2}^{2}\right)\right)}{\beta \lambda}}} \pm \frac{\sqrt{\frac{18\left(\mu^{2}-\lambda^{2}\left(C_{1}^{2}+C_{2}^{2}\right)\right)}{\beta \lambda}}}{C_{1} \sin (\sqrt{\lambda} \xi)+C_{2} \cos (\sqrt{\lambda} \xi)+\frac{\mu}{\lambda}},
$$


where, $\beta \neq 0$ and $\xi=x+y+z+\frac{3 \lambda\left(\mu^{2}+2 \lambda^{2}\left(C_{1}^{2}+C_{2}^{2}\right)\right)}{2\left(\lambda^{2}\left(C_{1}^{2}+C_{2}^{2}\right)-\mu^{2}\right)} t$.

In particular, if we set $C_{1}=0, C_{2} \neq 0, \beta<0$ and $\mu=0$ in Equation (61), then we get periodic solutions in the following form:

$$
u_{1}(\xi)= \pm \frac{\sqrt{\frac{-18 \lambda^{2} C_{2}^{2}}{\beta \lambda}}}{C_{2}} \sec (\sqrt{\lambda} \xi) .
$$

However, if we set $C_{1} \neq 0, C_{2}=0, \beta<0$ and $\mu=0$ in Equation (61), then we get periodic solutions in the following form:

$$
u(\xi)= \pm \frac{\sqrt{\frac{-18 \lambda^{2} C_{1}^{2}}{\beta \lambda}}}{C_{1}} \operatorname{cosec}(\sqrt{\lambda} \xi) .
$$

The obtained solutions given by (61) and (62) are presented in Figure 1 and Figure 2, respectively.

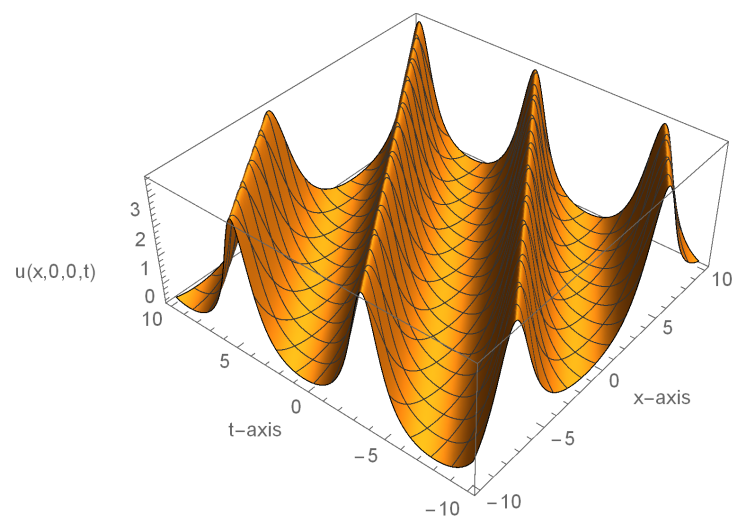

Figure 1. Graphical representation of Case 1 for the (3+1)-dimensional modified KdV-ZakharovKuznetsov equation when $C_{1}=C_{2}=1, \beta=1, \mu=0.5, \lambda=0.25$ within the interval $-10 \leq x, t \leq 10$.

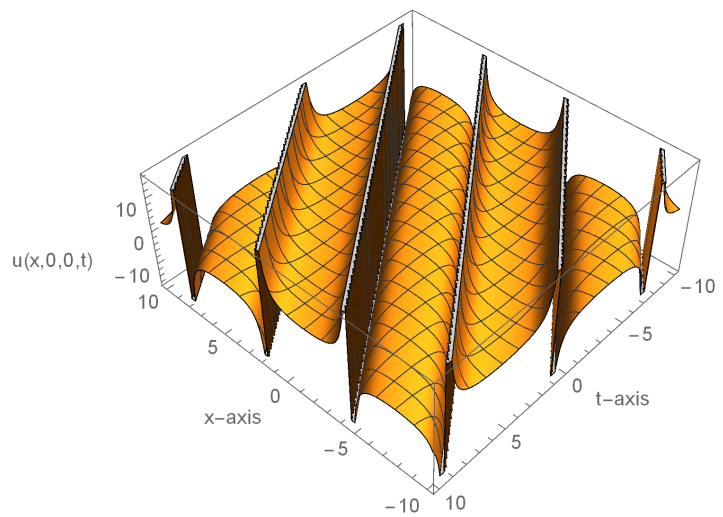

Figure 2. Graphical representation of Case 1 for the (3+1)-dimensional modified KdV-ZakharovKuznetsov equation when $C_{1}=0, C_{2}=1, \beta=-1, \mu=0, \lambda=0.25$ within the interval $-10 \leq x, t \leq 10$.

Case 2 (Hyperbolic function solutions) $\lambda<0$.

Substituting Equation (60) into Equation (59) along with Equations (10)-(12) and collecting coefficients of polynomials of $\phi^{i} \gamma^{j}(i, j=0,1,2,3)$, we obtain an algebraic system of $\rho, \mu, \lambda, \beta, a_{0}, a_{1}$, and $b_{0}$. Then, we solve these system via the Maple packet program, and we obtain 


$$
\begin{array}{ccrl}
a_{0} & =\mp \frac{9 \mu}{\beta \sqrt{\frac{18\left(\mu^{2}+\lambda^{2} \rho\right)}{\beta \lambda}}}, \quad a_{1}=0, & b_{1}= \pm \sqrt{\frac{18\left(\mu^{2}+\lambda^{2} \rho\right)}{\beta \lambda}}, \\
v=\frac{3 \lambda\left(\mu^{2}-2 \lambda^{2} \rho\right)}{2\left(\mu^{2}+\lambda^{2} \rho\right)}, & C= \pm \frac{27 \lambda^{3} \rho \mu}{\left(\mu^{2}+\lambda^{2} \rho\right) \beta \sqrt{\frac{18\left(\mu^{2}+\lambda^{2} \rho\right)}{\beta \lambda}}} .
\end{array}
$$

If we substitute these results into Equation (60) along with Equations (9) and (12), we obtain the exact solutions of the (3+1)-dimensional modified KdV-Zakharov-Kuznetsov equation as follows:

$$
u(\xi)=\mp \frac{9 \mu}{\beta \sqrt{\frac{18\left(\mu^{2}+\lambda^{2}\left(C_{1}^{2}-C_{2}^{2}\right)\right)}{\beta \lambda}}} \pm \frac{\sqrt{\frac{18\left(\mu^{2}+\lambda^{2}\left(C_{1}^{2}-C_{2}^{2}\right)\right)}{\beta \lambda}}}{C_{1} \sinh (\sqrt{-\lambda} \xi)+C_{2} \cosh (\sqrt{-\lambda} \xi)+\frac{\mu}{\lambda}}
$$

where $\beta \neq 0$ and $\xi=x+y+z+\frac{3 \lambda\left(\mu^{2}-2 \lambda^{2}\left(C_{1}^{2}-C_{2}^{2}\right)\right)}{2\left(\lambda^{2}\left(C_{1}^{2}-C_{2}^{2}\right)+\mu^{2}\right)} t$.

In particular, if we set $C_{1}=0, C_{2} \neq 0, \beta>0$ and $\mu=0$ in Equation (64), then we obtain the solitary wave solutions in the following form:

$$
u(\xi)= \pm \frac{\sqrt{\frac{-18 \lambda^{2} C_{2}^{2}}{\beta \lambda}}}{C_{2}} \operatorname{sech}(\sqrt{-\lambda} \xi) .
$$

In contrast, if we set $C_{1} \neq 0, C_{2}=0, \beta<0$ and $\mu=0$ in Equation (64), then we obtain the solitary wave solutions in the following form.

$$
u(\xi)= \pm \frac{\sqrt{\frac{18 \lambda^{2} C_{1}^{2}}{\beta \lambda}}}{C_{1}} \operatorname{cosech}(\sqrt{-\lambda} \xi)
$$

The obtained solutions given by (65) and (66) are presented in Figure 3 and Figure 4, respectively.

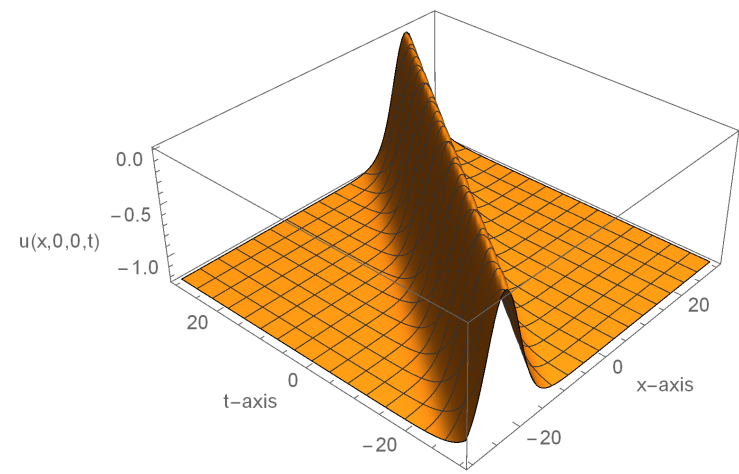

Figure 3. Graphical representation of Case 2 for the (3+1)-dimensional modified KdV-ZakharovKuznetsov equation when $C_{1}=0, C_{2}=1, \beta=-1, \mu=-0.5, \lambda=-0.25$ within the interval $-30 \leq x, t \leq 30$. 


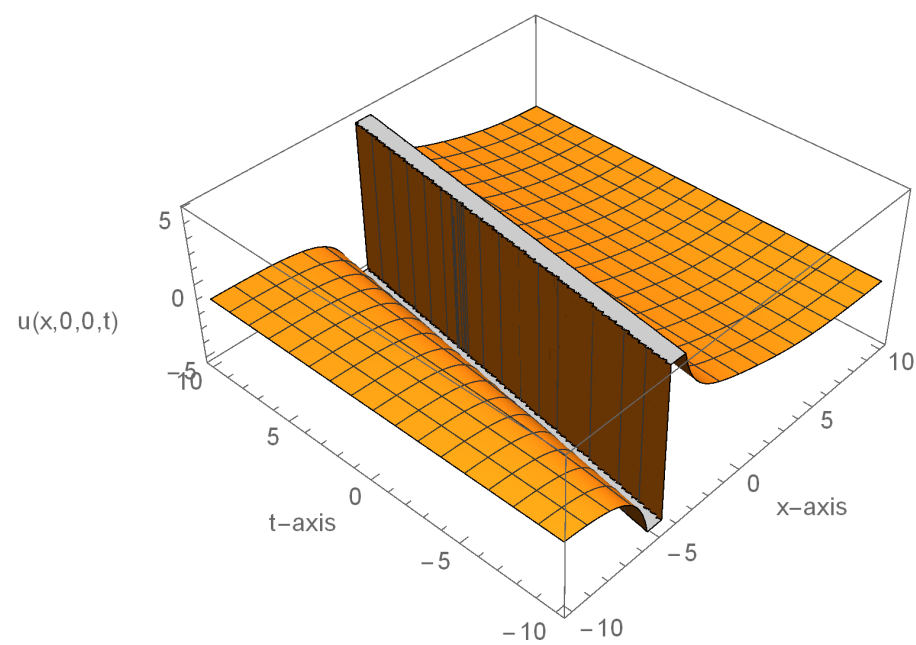

Figure 4. Graphical representation of Case 2 for the $(3+1)$-dimensional modified KdV-ZakharovKuznetsov equation when $C_{1}=1, C_{2}=0, \beta=-1, \mu=-0.5, \lambda=-0.25$ within the interval $-10 \leq x, t \leq 100$.

Case 3 (Rational function solutions) $\lambda=0$.

Substituting Equation (60) into Equation (59) along with Equations (10)-(13) and collecting coefficients of polynomials of $\phi^{i} \gamma^{j}(i, j=0,1,2,3)$, we obtain an algebraic system of $\rho, \mu, \lambda, \beta, a_{0}, a_{1}, b_{0}$. Then, we solve these system via the Maple packet program, and we find the following:

$$
\begin{gathered}
a_{0}=\mp \frac{9 \mu}{\beta \sqrt{\frac{18\left(2 \mu C_{2}-C_{1}^{2}\right)}{\beta}}, \quad a_{1}=0,} \quad b_{1}= \pm \sqrt{\frac{18\left(2 \mu C_{2}-C_{1}^{2}\right)}{\beta}}, \\
v=\frac{9 \mu^{2}}{2\left(2 \mu C_{2}-C_{1}^{2}\right)},
\end{gathered}
$$

If we substitute these results into Equation (60) along with Equations (9) and (13), we obtain the exact solutions of the (3+1)-dimensional modified KdV-Zakharov-Kuznetsov equation as follows:

$$
u(\xi)=\mp \frac{9 \mu}{\beta \sqrt{\frac{18\left(2 \mu C_{2}-C_{1}^{2}\right)}{\beta}}} \pm \frac{\sqrt{\frac{18\left(2 \mu C_{2}-C_{1}^{2}\right)}{\beta}}}{\frac{\mu}{2} \xi^{2}+C_{1} \xi+C_{2}}
$$

where $\beta \neq 0$ and $\xi=x+y+z+\frac{9 \mu^{2}}{2\left(C_{1}^{2}-2 \mu C_{2}\right)} t$.

In particular, if we set $c_{1}=0$ and $c_{2} \neq 0$ in Equation (67), then we get the solitary wave solutions in the following form:

$$
u(\xi)=\mp \frac{9 \mu}{\beta \sqrt{\frac{36 \mu C_{2}}{\beta}}} \pm \frac{\sqrt{\frac{136 \mu C_{2}}{\beta}}}{\frac{\mu}{2} \xi^{2}+C_{2}} .
$$

In contrast, if we set $c_{1} \neq 0$ and $c_{2}=0$ in Equation (67), then we obtain the solitary wave solutions in the following form:

$$
u(\xi)=\mp \frac{9 \mu}{\beta \sqrt{\frac{-18 C_{1}^{2}}{\beta}}} \pm \frac{\sqrt{\frac{-18 C_{1}^{2}}{\beta}}}{\frac{\mu}{2} \xi^{2}+C_{1} \xi} .
$$


When we compare our solutions with the other solutions in the literature, we can state that our solutions are new and more extensive than the given results in [43]. Furthermore, our solutions are comparable with those in $[44,45]$. We foresee that the obtained results will be useful for applications in mathematical physics and engineering. All results in this paper were found and checked with the help of Maple software.

\section{Conclusions}

In this study, we considered the (3+1)-dimensional modified KdV-Zakharov-Kuznetsov equation. We used the conservation theorem method to obtain the local conservation laws of the given equation. We obtained the formal Lagrangian; then, we found the adjoint equation by applying the formal Lagrangian to the variational derivative. It was seen that modified KdV-Zakharov-Kuznetsov equation is self adjoint. Using the solution of the adjoint equation, we obtained 12 modified local conservation laws. The two-variable $\left(G^{\prime} / G, 1 / G\right)$-expansion method, which was used to obtain exact solutions of NPDEs, can be considered as a generalization of the original $\left(G^{\prime} / G\right)$-expansion method. Furthermore, graphical representation is a significant instrument for communication, and it clearly presents the solutions to the problems. We plotted solutions of the $(3+1)$-dimensional modified $\mathrm{KdV}$-Zakharov-Kuznetsov equation.

We consider that the proposed method can be extended to solve the nonlinear problems which arise in mathematical physics and the theory of solitons. We successfully applied this method to the (3+1)-dimensional modified KdV-Zakharov-Kuznetsov equation. The obtained solutions are classified as trigonometric function solutions, hyperbolic function solutions and rational function solutions. We foresee that our results will be useful for applications in mathematical physics and engineering.

Author Contributions: A.A. and H.A., methodology; M.K. and M.S.O., software; M.K., H.A. and A.A., formal analysis; M.K., M.S.O. and H.A., writing-review and editing. All authors have read and agreed to the published version of the manuscript.

Funding: Not applicable.

Institutional Review Board Statement: Not applicable.

Informed Consent Statement: Not applicable.

Data Availability Statement: Data sharing is not applicable to this article as no data sets were generated or analyzed during the current study.

Conflicts of Interest: The authors declare no conflict of interest.

\section{References}

1. Osman, M.S.; Rezazadeh, H.; Eslami, M.; Neirameh, A.; Mirzazadeh, M. Analytical study of solitons to benjamin-bona-mahonyperegrine equation with power law nonlinearity by using three methods. U. Politeh. Buch. Ser. A 2018, 80, 267-278.

2. Ali, K.K.; Cattani, C.; Gómez-Aguilar, J.F.; Baleanu, D; Osman, M.S.; Analytical and numerical study of the DNA dynamics arising in oscillator-chain of Peyrard-Bishop model. Chaos Soliton. Fract. 2020, 139, 110089. [CrossRef]

3. Liu, J.G.; Osman, M.S.; Wazwaz, A.M. A variety of nonautonomous complex wave solutions for the (2+1)-dimensional nonlinear Schrödinger equation with variable coefficients in nonlinear optical fibers. Optik 2019, 180, 917-923. [CrossRef]

4. Osman, M.S. Multi-soliton rational solutions for quantum Zakharov-Kuznetsov equation in quantum magnetoplasmas. Waves Random Complex Media 2016, 26, 434-443. [CrossRef]

5. Hirota, R. Direct Method of Finding Exact Solutions of Nonlinear Evolution Equations; Bullough, R., Caudrey, P., Eds.; Backlund Transformations; Springer: Berlin, Germany, 1980.

6. Osman, M.S.; Inc, M.; Liu, J.G.; Hosseini, K.; Yusuf, A. Different wave structures and stability analysis for the generalized (2+1)-dimensional Camassa-Holm-Kadomtsev-Petviashvili equation. Phys. Scr. 2020, 95, 035229. [CrossRef]

7. Wazwaz, A.M. Multiple soliton solutions for (2+1)-dimensional Sawada-Kotera and Caudrey-Dodd-Gibbon equations. Math. Meth. Appl. Sci. 2011, 34, 1580-1586. [CrossRef]

8. Liu, J.G.; Zhu, W.H.; Osman, M.S.; Ma, W.X. An explicit plethora of different classes of interactive lump solutions for an extension form of 3D-Jimbo-Miwa model. Eur. Phys. J. Plus 2020, 135, 412. [CrossRef]

9. Yusufoğlu, E.; Bekir, A. Exact solutions of coupled nonlinear evolution equations. Chaos Solitons Fractals 2008, 37, 842-848. [CrossRef] 
10. Misirli, E.; Gurefe, Y. Exp-function method to solve the generalized Burgers-Fisher equation. Nonlinear Sci. Lett. A 2010, 13, 323-328.

11. Zhang, S. Application of Exp-function method to a KdV equation with variable coefficients. Phys. Lett. A 2007, 365, 448-453. [CrossRef]

12. Naher, H. New approach of $\left(G^{\prime} / G\right)$-expansion method and new approach of generalized $\left(G^{\prime} / G\right)$-expansion method for ZKBBM equation. J. Egypt. Math. Soc. 2015, 23, 42-48. [CrossRef]

13. Ding, Y.; Osman, M.S.; Wazwaz, A.M. Abundant complex wave solutions for the nonautonomous FokasLenells equation in presence of perturbation terms. Optik 2019, 181, 503-513. [CrossRef]

14. Zayed, E.M.E.; Al-Joudi, S. Applications of an Extended $\left(G^{\prime} / G\right)$-Expansion Method to Find Exact Solutions of Nonlinear PDEs in Mathematical Physics. Math. Probl. Eng. 2010, 2010, 19. [CrossRef]

15. Alam, M.N.; Bonyah, E.; Fayz-Al-Asad, M.; Osman, M.S.; Abualnaja, K.M. Stable and functional solutions of the Klein-FockGordon equation with nonlinear physical phenomena. Phys. Scr. 2021, 96, 055207. [CrossRef]

16. Gurefe, Y.; Misirli, E.; Sonmezoglu, A.; Ekici, M. Extended trial equation method to generalized partial differential equations. Appl. Math. Comput. 2013, 219, 5253-5260. [CrossRef]

17. Tascan, F.; Bekir, A.; Koparan, M. Travelling wave solutions of nonlinear evolution equations by using the first integral method. Commun. Nonlinear Sci. Numer. Simul. 2009, 14, 1810-1815. [CrossRef]

18. Wazwaz, A.M. A sine-cosine method for handling nonlinear wave equations. Math. Comput. Model. 2004, 40, 499-508. [CrossRef]

19. Kaplan, M.; Akbulut, A.; Bekir, A. Exact travelling wave solutions of the nonlinear evolution equations by auxiliary equation method. Z. Naturforsch. A 2015, 70, 969-974. [CrossRef]

20. Naz, R.; Mahomed, F.M.; Mason, D.P. Conservation laws via the partial Lagrangian and invariant solutions for radial and two-dimensional free jets. Nonlinear Anal. Real World Appl. 2009, 10, 3457-3465. [CrossRef]

21. Bluman, G.W.; Kumei, S. Symmetries and Differential Equations with 21 Illustrations; Springer-Verlag: New York, NY, USA, 1989.

22. Ibragimov, N.H.; Khamitova, R.; Avdonina, E.D.; Galiakberova, L.R. Conservation laws and solutions of a quantum drift-diffusion model for semiconductors. Int. J. Non-Linear Mech. 2015, 77, 69-73. [CrossRef]

23. Olver, P.J. Application of Lie Groups to Differential Equations; Springer-Verlag: New York, NY, USA, 1993.

24. Ibragimov, N.H. A new conservation theorem. J. Math. Anal. Appl. 2007, 333, 311-328. [CrossRef]

25. Anco, S.C.; Bluman, G.W. Direct construction method for conservation laws of partial differential equations. Part II: General treatment. Eur. J. Appl. Math. 2002, 9, 567-585. [CrossRef]

26. Gandarias, M.L. Conservation laws for some equations that admit compacton solutions induced by a non-convex convection. J. Math. Anal. Appl. 2015, 430, 695-702. [CrossRef]

27. Tutueva, A.; Karimov, T.; Butusov, D. Semi-Implicit and Semi-Explicit Adams-Bashforth-Moulton Methods. Mathematics 2020, 8 , 780. [CrossRef]

28. Kress, W.; Lötstedt, P. Time step restrictions using semi-explicit methods for the incompressible Navier-Stokes equations. Comput. Method Appl. Mech. Eng. 2006, 195, 4433-4447. [CrossRef]

29. Bürger, R.; Gavilán, E.; Inzunza, D.; Mulet, P.; Villada, L.M. Implicit-explicit methods for a convection-diffusion-reaction model of the propagation of forest fires. Mathematics 2020, 8, 1034. [CrossRef]

30. Ibragimov, N.H.; Kolsrud, T. Lagrangian Approach to Evolution Equations: Symmetries and Conservation Laws. Nonlinear Dyn. 2004, 36, 29-40. [CrossRef]

31. Buhe, E.; Bluman, G.; Kara, A.H. Conservation laws for some systems of nonlinear PDEs via the symmetry/adjoint symmetry pair method. J. Math. Anal. Appl. 2016, 436, 94-103. [CrossRef]

32. Xin, X.; Liu, Y.; Liu, X. Nonlocal symmetries, exact solutions and conservation laws of the coupled Hirota equations. Appl. Math. Lett. 2016, 55, 63-71. [CrossRef]

33. Gandarias, M.L.; Khalique, C.M. Symmetries, solutions and conservation laws of a class of nonlinear dispersive wave equations. Commun. Nonlinear Sci. Numer. Simul. 2016, 32, 114-121. [CrossRef]

34. Osman, M.S.; Baleanu, D.; Adem, A.R.; Hosseini, K.; Mirzazadeh, M.; Eslami, M. Double-wave solutions and Lie symmetry analysis to the (2+1)-dimensional coupled Burgers equations. Chin. J. Phys. 2020, 63, 122-129. [CrossRef]

35. Kumar, S.; Kour, B.; Yao, S.W.; Inc, M.; Osman, M.S. Invariance Analysis, Exact Solution and Conservation Laws of (2+1) Dim Fractional Kadomtsev-Petviashvili (KP) System. Symmetry 2021, 13, 477. [CrossRef]

36. Tascan, F.; Yakut, A. Conservation Laws and Exact Solutions with Symmetry Reduction of Nonlinear Reaction Diffusion Equations Int. J. Nonlinear Sci. Numer. Simul. 2015, 16, 191-196. [CrossRef]

37. Yakut, A. Consevation Laws for Partial Differential Equations. Master's Thesis, Eskişehir Osmangazi University, Eskişehir, Turkey, 2012. [CrossRef]

38. Yaşar, E. Conservation laws for a class of soil water equations. Commun. Nonlinear Sci. Numer. Simul. 2010, 15, 3193-3200.

39. Li, L.X.; Li, E.Q.; Wang, M.L. The $\left(G^{\prime} / G, 1 / G\right)$-expansion method and its application to travelling wave solutions of the Zakharov equations. Appl. Math. J. Chin. Univ. 2010, 25, 454-462. [CrossRef]

40. Kaplan, M. Exact Solutions of Nonlinear Schrödinger Equation. Master's Thesis, Eskişehir Osmangazi University, Eskişehir, Turkey, 2013. [CrossRef]

41. Akbulut, A.; Kaplan, M.; Taşcan, F. Conservation laws and Exact Solutions of Phi-Four (Phi-4) Equation via the (G//G, 1/G)Expansion Method. Z. Naturforsch. A 2016, 71, 439-446. 
42. Alam, M.N.; Hafez, M.G.; Akbar, M.A.; Roshid, H.O. Exact traveling wave solutions to the (3+1)-dimensional mKdV-ZK and the (2+1)-dimensional Burgers equations via $\exp (-\Phi(\eta))$-expansion method. Alex. Eng. J. 2015, 54, 635-644. [CrossRef]

43. Khan, K.; Akbar, M.A. Exact and solitary wave solutions for the Tzitzeica-Dodd-Bullough and the modified KdV-ZakharovKuznetsov equations using the modified simple equation method. Ain Shams Eng. J. 2014, 4, 903-909. [CrossRef]

44. slam, M.H.; Khan, K.; Akbar, M.A.; Salam, M.A. Exact traveling wave solutions of modified KdV-Zakharov-Kuznetsov equation and viscous Burgers equation. SpringerPlus 2014, 3, 105. [CrossRef]

45. Naher, H.; Abdullah, F.A.; Akbar, M.A. Generalized and Improved ( $\left.G^{\prime} / G\right)$-Expansion Method for (3+1)-Dimensional Modified KdV-Zakharov-Kuznetsov Equation. PLoS ONE 2013, 8, e64618. 